\title{
Development of a Kinect Rehabilitation System
}

\author{
http://dx.doi.org/10.3991/ijoe.v9iS8.3378 \\ João Couto Soares ${ }^{1}$, Ágata Vieira ${ }^{2}$, Octavian Postolache ${ }^{3,4}$ and Joaquim Gabriel ${ }^{1}$ \\ ${ }^{1}$ IDMEC Pólo FEUP, Faculty of Engineering, University of Porto, Porto, Portugal \\ 2 ICBAS, Abel Salazar Biomedical Sciences Institute, University of Porto, Porto, Portugal \\ ${ }^{3}$ ISCTE-IUL, IT-IUL, University Institute of Lisbon, Lisbon, Portugal \\ ${ }^{4}$ IT-IST, DEEC-IST, Instituto Superior Técnico, Lisbon, Portugal
}

\begin{abstract}
Microsoft Kinect camera has been used in serious games applications, like for rehabilitation purposes, almost since it became available in the market. This article presents a clinical view regarding home-based physiotherapy for patients that suffered a stroke and details on the development of the rehabilitation system - KinectRehabPlay. This system uses the Kinect sensor together with the Unity3D game engine software to create the animation and visual environment. Currently, it is able to track, recording and comparing movements (doctor versus patient), and adjust the game configuration in real-time.
\end{abstract}

Index Terms - kinect, serious games, stroke rehabilitation.

\section{INTRODUCTION}

As the world population is getting older, elders represent a large percentage, especially in Europe and Japan, where the birthrate decrease is a serious social, political and economic issue [1]. Additionally, the number of people prone to a high risk of cardiac diseases is also increasing, mainly due to stress, a low exercise live style, unhealthy food and smoking habits. On the other hand, current cardiac rehabilitation health programs are expensive [2] and force patients to miss working time to go to rehabilitation centers for supervised training. Therefore, the development of a home assisted rehabilitation program based on games technology seems to be a good approach to address this issue.

This document presents a home-based rehabilitation system making use of the Kinect sensor, from Microsoft.

\section{CurRent Systems}

Kinect for Xbox ${ }^{1}$ is a low cost gaming peripheral composed of several sensors that is able to act as a tracking device. In June of 2011, Microsoft released a software development kit (SDK) that allowed the linkage between the sensors and a computer. Later, in February 2012, a new variant of the sensor was released, the Kinect for Windows ${ }^{2}$, specifically for Windows computer applications.

The use of this sensor for rehabilitation solutions in clinical and non-clinical environments, such as schools or at home, has been object of experimentation for several health afflictions since its debut and several studies of comparison between the Kinect sensor and other motion capture (MOCAP) devices have been carried out, validating the Kinect sensor measures.

http://www.xbox.com/kinect

http://www.kinectforwindows.com
In reference [3], the Kinect sensor is presented as providing an "acceptable level of quality of tracking performance". Reference [4] makes a comparison between the process of skeletonization (pose measurement) provided by the Kinect sensor and a fixed MOCAP system, concluding that it is able to correctly detect a "controlled body posture (e.g. standing and exercising arms)". The Kinect sensor's angle joints measurement accuracy is also validated for most of the prescribed exercises in rehabilitation treatments by reference [5].

Lately, several projects that seek to solve the access to rehabilitation of several different health issues have been presented. In reference [6], the Kinect sensor is used in a hand rehabilitation scenario. The Kinect sensor was also used for full body gait analysis by reference [7], which can be applied for a wide range of diseases, such as diabetes and neurological conditions, but also for fall detection and prediction of such occurrence. A study of fall risk assessment and cognitive impairment was also conducted using a Kinect sensor in reference [8].

The association of physical rehabilitation and serious games fields is fundamental for home-based rehabilitation system solutions. In reference [9], a public school setting was used for a study on a motor disabilities rehabilitation game (fine motor skills, strength and range of motion training). When the users performed as expected, a singing whale with its tail flipping on the surface of the sea would appear on the game screen. Spinal cord injury rehabilitation games were proposed in [3], [10], [11]. The first game placed the user in a mine cart and the aim of the user was to gather gems and place them in the cart as he/she travelled through the mines. The second game encourages the user to use his/her hand to move a virtual object from one side of the screen to the other. For stroke rehabilitation, an upper limb game training approach was presented by reference [12]. In it, the users are asked to reach for and to catch a virtual ball by controlling an avatar. Reference [13] also presents two approaches regarding stroke rehabilitation, but using both hands and feet. In one game, the user has to control an avatar in order to climb a hill, and on the other, control an avatar to run. For stroke rehabilitation as well, reference [14] presents a game for upper limbs rehabilitation, in which the Kinect sensor is mounted facing down, so it can measure the range of motion and speed of movement of the user's arms.

\section{Clinical Point Of View Of The Problem}

Mortality, economic burden and morbidity worldwide are greatly increased by cardiovascular diseases (CVDs) [2]. Most health concerns would be reduced if CVDs 
potential patients engaged in cardiovascular rehabilitation programs [2]. These exercise-based programs are patienttailored, aiming to improve the quality of life, reduce mortality, decrease disability, reduce costs of treatment and restore and improve cardiac function [15].

As most of the programs for cardiovascular rehabilitation are limited to institutions in large urban areas, accessibility is the main limitation of the traditional CVDs rehabilitation solutions. In Europe, only a third of coronary heart disease patients receive support for cardiovascular rehabilitation, and most programs are of short-term [16], in case of being implemented. Furthermore, this kind of programs' costs are often beyond the monetary resources of most patients.

The implementation of cardiovascular home-based programs (home context) is a possible solution for this lack of service. The passage of cardiovascular rehabilitation programs to the community, especially for the home context, self-blaming the patient and privileging the empowerment and health is suggested by the current scientific evidence [17]. The physical activity outcome, appears to be of little or no difference when comparing home rehabilitation with comprehensive institutionalizedbased care, suggesting that both approaches to cardiac rehabilitation are effective and that there is no difference between the two [18].

As new technologies contribute to an increase of innovative intervention strategies (which may contribute to increase the motivation and predisposition of individuals for their rehabilitation process), the association of physical rehabilitation and serious games fields is a fundamental piece for home-based rehabilitation system solutions [19], [20]. For those patients who are unable or unwilling to attend local cardiovascular rehabilitation programs, the virtual based cardiovascular programs may be a viable alternative. The use of technology to deliver health-care has been suggested as a potential solution to reach patients at a distance [21].

\section{THE KINECT-REHABPLAY SYSTEM}

Analyzing the current method of rehabilitation for cardiac afflictions, which requires physical presence at the clinic and the associated costs, a solution based in serious games for home assisted rehabilitation seems to be more convenient for the patients and caregiver, and also in economic terms.

The Kinect-RehabPlay project makes use of software to monitor and evaluate the rehabilitation exercises prescribed by a certified physical therapist, which have to be executed by the user and captured by the Kinect sensor, providing him/her with real time results about the given task.

\section{A. Objectives}

The primary aim of the project is to provide a low cost system for home use, which permits persons with cardiac rehabilitation necessities to be able to perform the proposed rehabilitation exercises without the need to leave home and at their preferred schedule.

\section{B. Architecture}

The Kinect-RehabPlay system is composed by three modules: the virtual reality environment, the Kinect sensor and the monitoring software package. The system is presented in Fig. 1.

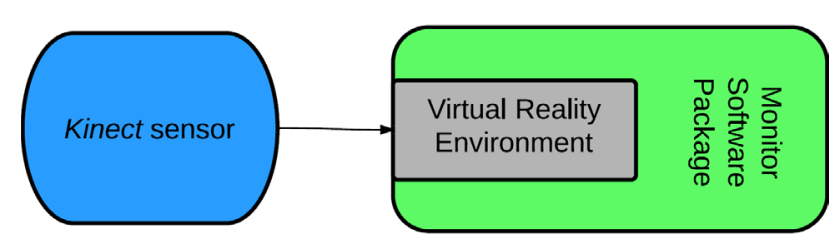

Figure 1. The Kinect-RehabPlay system

As shown, the Kinect sensor is used as an input to the virtual reality environment, which in turn is monitored by the monitoring software package. In the following sections, a brief description about each of the modules is presented.

\section{1) Virtual Reality Environment}

As the project objective is to develop a game for rehabilitation purposes, the virtual environment in which it takes place is a significant part of the Kinect-RehabPlay system.

Besides graphically presenting the exercises to execute, it should also help to motivate the user to continue and to perform accordingly as indicated, so that the user does not hurt himself/herself.

Snapshots of two game levels are displayed in Fig. 2a (beach scenery) and Fig. 2b (Paris background scenery).

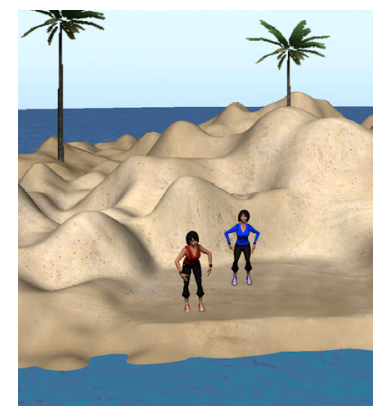

(a) Beach scenery

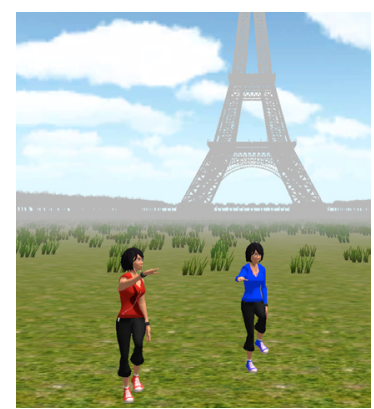

(b) Paris scenery
Figure 2. Game levels

The game level's scenery presents two avatars, one representing the physiotherapist and the other avatar on the scene is controlled by the user's movements, making use of the Kinect sensor.

\section{2) Kinect sensor}

Acting as the only input to the system, the Kinect sensor is of great importance. It allows to interpret the rehabilitation positions performed by the user, to control the user avatar and it also delivers part of the data to the monitoring software package.

The prototype currently makes use of SDK version $1.7^{3}$, released on March of 2013. The SDK is able to provide the system with points that represent the users' joints. The points are calculated using a machine-learning algorithm, making sure the Kinect sensor can measure any kind of body, size and postures [22].

http://www.microsoft.com/enus/download/details.aspx?id=36996 


\section{3) Monitoring Software Package}

This module essentially acts as a monitor to the virtual reality environment, but it can also perform on it, triggering motivation notices or warnings (regarding incorrect positioning by the user and possible injuries), but also increasing or decreasing the game difficulty level and speed, according to the user's performed movements and health condition.

The check to whether the user is performing the exercises as he/she should, without injuring himself/herself, is executed by controlling the angle of the joints provided by the Kinect sensor, together with a comparison with the movements previously recorded by a physiotherapist.

\section{CONClusions}

The current system, which still under development, is expected to help in home-based rehabilitation, by assisting one's individual exercises in a standalone fashion. At the moment, it can record the physiotherapist movements and reproduce them at a variable speed. Also, it can follow and record the users' movement for comparison.

The system is programmed in a way that can be easily adapt to be applied to other diseases that can take advantage from the use of rehabilitation for its treatment, provide even broader functionality to the system.

\section{A. Future Developments}

In order to extend the functionality and usability of the system, it is planned to add voice command support and also the ability to connect to various types of health monitoring sensors (e.g. heartbeat measurement).

\section{REFERENCES}

[1] U. N. D. of Economic and S. A. D. P. Division, "World Population Prospects: The 2010 Revision," Department of Economics and Social Affairs, Population Division, New York, 2011.

[2] H. S. Buttar, T. Li, and N. Ravi, "Prevention of cardiovascular diseases: Role of exercise, dietary interventions, obesity and smoking cessation.," Exp. Clin. Cardiol., vol. 10, no. 4, pp. 229 $249,2005$.

[3] C. Y. Chang et al., "Towards Pervasive Physical Rehabilitation Using Microsoft Kinect," pp. 159-162, 2012.

[4] S. Obdrzalek et al., "Accuracy and robustness of Kinect pose estimation in the context of coaching of elderly population," in Engineering in Medicine and Biology Society (EMBC), 2012 Annual International Conference of the IEEE, 2012, pp. 11881193.

[5] A. Fernández-Baena, A. Susin, and X. Lligadas, "Biomechanical Validation of Upper-Body and Lower-Body Joint Movements of Kinect Motion Capture Data for Rehabilitation Treatments," in Intelligent Networking and Collaborative Systems (INCOS), 2012 4th International Conference on, 2012, pp. 656-661.

[6] F. Cordella, F. Di Corato, L. Zollo, B. Siciliano, and P. Van Der Smagt, "Patient performance evaluation using Kinect and Monte Carlo-based finger tracking," in Biomedical Robotics and Biomechatronics (BioRob), 2012 4th IEEE RAS EMBS International Conference on, 2012, pp. 1967-1972.

[7] M. Gabel, R. Gilad-Bachrach, E. Renshaw, and A. Schuster, "Full body gait analysis with Kinect.," Conf. Proc. IEEE Eng. Med. Biol. Soc., vol. 2012, pp. 1964-7, Jan. 2012.

[8] E. E. Stone and M. Skubic, "Capturing habitual, in-home gait parameter trends using an inexpensive depth camera," in Engineering in Medicine and Biology Society (EMBC), 2012 Annual International Conference of the IEEE, 2012, pp. 51065109 .
[9] Y. J. Chang, S. F. Chen, and J. D. Huang, “A Kinect-based system for physical rehabilitation: a pilot study for young adults with motor disabilities.," Res. Dev. Disabil., vol. 32, no. 6, pp. 256670. http://dx.doi.org/10.1016/j.ridd.2011.07.002

[10] B. Lange et al., "Development and evaluation of low cost gamebased balance rehabilitation tool using the microsoft kinect sensor," in Engineering in Medicine and Biology Society,EMBC, 2011 Annual International Conference of the IEEE, 2011, pp. 1831-1834.

[11] B. Lange et al., "Interactive game-based rehabilitation using the Microsoft Kinect," in Virtual Reality Short Papers and Posters (VRW), 2012 IEEE, 2012, pp. 171-172.

[12] S. C. Yeh et al., "A Study for the Application of Body Sensing in Assisted Rehabilitation Training," 2012 Int. Symp. Comput. Consum. Control, pp. 922-925, Jun. 2012. http://dx.doi.org/10.1109/IS3C.2012.240

[13] S. Saini et al., "A Low-cost Game Framework for a Home-based Stroke Rehabilitation System," pp. 55-60, 2012.

[14] I. Pastor, H. A. Hayes, and S. J. M. Bamberg, "A feasibility study of an upper limb rehabilitation system using kinect and computer games," in Engineering in Medicine and Biology Society (EMBC), 2012 Annual International Conference of the IEEE, 2012, pp. 1286-1289.

[15] A. M. and F. Rosa, "O síndrome coronário agudo nos cuidados de saúde primários.,"Acta Med. Port., vol. 23, no. 2, 2010.

[16] L. F. Hamm et al., "Core competencies for cardiac rehabilitation/secondary prevention professionals: 2010 update: position statement of the American Association of Cardiovascular and Pulmonary Rehabilitation," J Cardiopulm Rehabil Prev, vol. 31, no. 1, pp. 2-10, 2011.

[17] C. L. Huang and H. H. Wang, "Community health development: what is it?," Int Nurs Rev, vol. 52, no. 1, pp. 13-17, 2005. http://dx.doi.org/10.1111/j.1466-7657.2004.00259.x

[18] J. Blair, H. Corrigall, N. J. Angus, D. R. Thompson, and S. Leslie, "Home versus hospital-based cardiac rehabilitation: a systematic review.," Rural Remote Heal., vol. 11, no. 2, p. 1532, 2011.

[19] P. J. Groen and D. Goldstein, "Gaming Technology, Virtual Reality and Healthcare." Apr-2008.

[20] P. Marshall et al., "Randomized controlled trial of home-based exercise training to evaluate cardiac functional gains.," Clin Sci, vol. 101, no. 5, pp. 477-483, 2001.

[21] A. Zutz, A. Ignaszewski, J. Bates, and S. A. Lear, "Utilization of the internet to deliver cardiac rehabilitation at a distance: a pilot study.,"Telemed J E Heal., vol. 13, no. 3, pp. 323-330, 2007.

[22] J. Shotton et al., "Real-time Human Pose Recognition in Parts from Single Depth Images," in Proceedings of the 2011 IEEE Conference on Computer Vision and Pattern Recognition, 2011, pp. 1297-1304.

\section{AUTHORS}

João Couto Soares is with the Faculty of Engineering, University of Porto, Portugal (e-mail: coutosoares@fe.up.pt).

Ágata Vieira is with Abel Salazar Biomedical Sciences Institute, University of Porto, Portugal (e-mail: agatavieira78@gmail.com).

Octavian Postolache is with the University Institute of Lisbon and Instituto Superior Técnico, Portugal (e-mail: octavian.postolache@gmail.com).

Joaquim Gabriel is with IDMEC at the Faculty of Engineering, University of Porto, Portugal (e-mail: jgabriel@fe.up.pt).

This work was supported by Fundos FEDER through the Programa Operacional Factores de Competitividade - COMPETE and by Fundos Nacionais through FCT - Fundação para a Ciência e a Tecnologia through the project FCOMP-01-0124-FEDER-13852 and PTDC/DTPDES/1661/2012. This article is an extended and modified version of a paper presented at the International Conference exp.at'13, held 18-20 September 2013, in Coimbra, Portugal. Submitted 18 November 2013. Published as re-submitted by the authors 04 December 2013. 